\title{
Neoadjuvant chemotherapy in breast cancer: early response prediction with quantitative MR imaging and spectroscopy
}

\author{
DJ Manton*,', A Chaturvedi', A Hubbard ${ }^{3}$, MJ Lind', M Lowry', A Maraveyas', MD Pickles', DJ Tozer ${ }^{4}$ and \\ LW Turnbull'
}

'The Postgraduate Medical Institute of the University of Hull Division of Cancer in association with the Hull York Medical School, The University of Hull, Hull, East Yorkshire HU6 7RX, UK; ${ }^{2}$ The Department of Clinical Oncology, Hull and East Yorkshire Hospitals NHS Trust, The Princess Royal Hospital, Saltshouse Road, Hull, East Yorkshire HU8 9HE, UK; ${ }^{3}$ The Breast Screening Unit, Hull and East Yorkshire Hospitals NHS Trust, Castle Hill Hospital, Castle Road, Cottingham, East Yorkshire HUI6 5JQ, UK; ${ }^{4}$ NMR Research Unit, Institute of Neurology, Queen Square, London WCIN 3BG, UK

A prospective study was undertaken in women undergoing neoadjuvant chemotherapy for locally advanced breast cancer in order to determine the ability of quantitative magnetic resonance imaging (MRI) and proton spectroscopy (MRS) to predict ultimate tumour response (percentage decrease in volume) or to detect early response. Magnetic resonance imaging and MRS were carried out before treatment and after the second of six treatment cycles. Pharmacokinetic parameters were derived from $T_{1}$-weighted dynamic contrast-enhanced MRI, water apparent diffusion coefficient (ADC) was measured, and tissue water: fat peak area ratios and water $T_{2}$ were measured using unsuppressed one-dimensional proton spectroscopic imaging (30 and I 35 ms echo times). Pharmacokinetic parameters and ADC did not detect early response; however, early changes in water: fat ratios and water $T_{2}$ (after cycle two) demonstrated substantial prognostic efficacy. Larger decreases in water $T_{2}$ accurately predicted final volume response in $69 \%$ of cases ( $1 / / 6)$ while maintaining 100\% specificity and positive predictive value. Small/absent decreases in water: fat ratios accurately predicted final volume non-response in 50\% of cases (3/6) while maintaining $100 \%$ sensitivity and negative predictive value. This level of accuracy might permit clinical application where early, accurate prediction of non-response would permit an early change to second-line treatment, thus sparing patients unnecessary toxicity, psychological morbidity and delay of initiation of effective treatment. British Journal of Cancer (2006) 94, 427-435. doi:I0.1038/sj.bjc.6602948 www.bjcancer.com (C) 2006 Cancer Research UK

Keywords: breast neoplasms; chemotherapy; magnetic resonance; MR; spectroscopy; contrast enhancement; diffusion study

Recent statistics confirm that breast cancer remains one of the most prevalent and serious forms of neoplastic disease in the UK. Breast cancer accounts for more than one in four female cancer cases and currently demonstrates age-standardised incidence and mortality rates of 117 and 31 per 100000 , respectively (Toms, 2003).

Some primary breast cancers are considered inoperable at diagnosis owing to their problematical location and size, with tumours greater than $3 \mathrm{~cm}$ in diameter being associated with an increased risk of disseminated disease. In such cases, neoadjuvant chemotherapy is routinely used before surgery to increase the chances of a successful outcome. Assessing tumour response to chemotherapy is crucial to patient management and is currently achieved by monitoring changes in tumour size using clinical examination backed up by longitudinal X-ray or ultrasound mammography. A poor response to the primary treatment regime usually prompts either change of chemotherapy regime or an early resort to surgery. Poor response might also require a greater degree of surgical intervention.

Repeated X-ray mammography (XRM) has several drawbacks: discomfort, radiation exposure, geometric distortion owing to

*Correspondence: Dr DJ Manton, MRI Centre, Hull Royal Infirmary, Anlaby Road, Hull HU3 2JZ, UK; E-mail: d.j.manton@.hull.ac.uk Received 10 June 2005; revised 17 October 2005; accepted 7 December 2005 compression and magnification render tumour volume measurements less accurate than magnetic resonance imaging (MRI), and tumour may be impossible to distinguish from dense glandular tissue and fibrosis. Magnetic resonance imaging has been shown to correspond better with pathological size measurement than XRM (Esserman et al, 1999; Drew et al, 2001) and to be less obscured by dense breast diseases. Ultrasound measurement is more accurate than XRM, but fails when the tumour to be measured is larger than the field of view or complex in shape. Magnetic resonance imaging is also more accurate than ultrasound at detecting small volume residual tumour.

However, even if tumour volume changes can be accurately assessed by MRI, they may manifest themselves later than changes in underlying tumour function such as vascular density or permeability (Wasser et al, 2003). Therefore, vascular or metabolic parameters might provide a more sensitive indicator of early tumour response, thus permitting individual treatment regimes to be adjusted more rapidly, and sparing patients unnecessary morbidity, expense and delay in initiation of effective treatment. Treatment-induced changes in metabolism can be investigated using both positron emission tomography (Byrne et al, 2004; Kumar and Alavi, 2004; Weber, 2005) and single photon emission computed tomography (Buscombe et al, 1997), but the clinical utility of such examinations is reduced by the need to limit repeated radiation doses.

Treatment-induced changes in tumour neovasculature can be assessed using dynamic contrast-enhanced MRI (DCE-MRI) and 
quantified using pharmacokinetic (PK) modelling (Padhani, 2002; Knopp et al, 2003). Diffusion-weighted MRI can be used to detect changes in the apparent diffusion coefficient (ADC) for tissue water associated with changes in tissue and intracellular structure (Zhao et al, 1996; Gallons et al, 1999; Ross et al, 2003; Moffat et al, 2005). Proton magnetic resonance spectroscopy (MRS) can be used to characterise breast lesions through differences in the ratio of fat and water signals (Chu et al, 1987; Sijens et al, 1988) or the intensity of signal from choline-containing compounds (Bradamante et al, 1988; Katz-Brull et al, 2002; Bolan et al, 2003; Jacobs et al, 2004; Jacobs et al, 2005). Both fat: water ratios (Jagannathan et al, 1998) and choline levels (Preul et al, 2000; Jagannathan et al, 2001; Schwarz et al, 2002; Meisamy et al, 2004) have also been used to monitor treatment-induced changes, and phosphorous MRS has been used to predict response using differences in the levels of phosphocholine or phosphomonoesters (Shukla-Dave et al, 2002a, b; Arias-Mendoza et al, 2004). Sodium MRI might also be useful in monitoring treatment response (Kline et al, 2000; Schepkin et al, 2005).

A prospective study was, therefore, undertaken whereby three of these techniques (DCE-MRI, ADC measurement and MRS fat:water ratios) were carried out in the same patients to determine in each technique the relative prognostic utility. The study hypothesis was that a combination of the quantitative data from the three techniques would lead to a synergistic increase in prognostic accuracy and, therefore, provide an accurate, reliable and noninvasive early indication of tumour response to chemotherapy, which could potentially have a positive influence on patient management.

\section{MATERIALS AND METHODS}

\section{Chemotherapy regime and relative timing of MRI}

All women who were scheduled to undergo neoadjuvant chemotherapy for primary inoperable locally advanced breast cancer were approached to join the study, which had received Local Research Ethics Committee approval (reference number 03/00/ 038). Longitudinal MRI was carried out using a $1.5 \mathrm{~T}$ Signa Advantage clinical MRI scanner (International General Electric, Milwaukee, WI, USA) using a dedicated bilateral breast coil (Machnet BV, Eelde, Netherlands). A standard dosage chemotherapy regime was used involving intravenous administration of epirubicin (bolus: $60 \mathrm{mg} \mathrm{m}^{-2}$ body surface area), cyclophosphamide (bolus: $600 \mathrm{mg} \mathrm{m}^{-2}$ ) and 5-fluorouracil (continuous infusion by pump: $200 \mathrm{mg} \mathrm{m}^{-2}$ per day). Good clinical practice, including checking patients' cardiac status before therapy if necessary, was adhered to throughout the study. Magnetic resonance imaging and MRS were carried out at three time points: before the first course of chemotherapy $\left(\mathrm{TP}_{0}\right)$, after the second course but no more than 55 days after the first course $\left(\mathrm{TP}_{2}\right)$ and after the final (generally sixth) course $\left(\mathrm{TP}_{\mathrm{F}}\right)$. Therapeutic response was confirmed after surgery by pathological examination.

\section{MR imaging and spectroscopy}

All the MRI data at each time point were acquired in a single imaging session with an average length of $1 \mathrm{~h}$. Dynamic contrastenhanced MRI was carried out first, during which gadopentetate dimeglumine (formerly known as Gd-DTPA; Schering Health Care, Burgess Hill, UK) was injected as a bolus at a dose of $0.1 \mathrm{mmol} \mathrm{kg}^{-1}$ body weight, using a two-dimensional, $T_{1}$-weighted multislice, fast RF spoiled gradient echo (FSPGR) sequence. In order to attain adequate tissue coverage in all cases, imaging was carried out in either the coronal $(30 \mathrm{~cm}$ field-of-view, FOV) or sagittal $(20 \mathrm{~cm}$ FOV $)$ planes $(256 \times 128$ matrix in all cases $)$ with five, seven or nine slices (4-9 mm thick, $2 \mathrm{~mm}$ gaps). Scan timing parameters, therefore, ranged as follows: $\mathrm{TR}=8.8-11.1 \mathrm{~ms}$; $\mathrm{TE}=4.2 \mathrm{~ms}$ fractional; flip $=30^{\circ}$; temporal resolution $=10.5$ $14.5 \mathrm{~s} ; 35$ time points per slice; scan time $=6 \mathrm{~min} 8 \mathrm{~s}$ to $8 \mathrm{~min}$ $28 \mathrm{~s}$. Dynamic contrast-enhanced MRI was preceded by proton density-weighted FSPGR imaging (TR/TE/flip $=120 \mathrm{~ms} / 4.2 \mathrm{~ms}$ fractional $/ 8^{\circ}$ ) to enable correction for differences in native tissue $T_{1}$.

A single region-of-interest (ROI) that best delimited the lesion present was drawn, by an experienced radiologist, for each DCEMRI study. In-house software (developed using the IDL language, Research Systems Inc., Boulder, CO, USA) was then used to measure the mean pixel signal intensity (SI) within the ROI for the proton density image $\left(\mathrm{SI}_{\mathrm{PD}}\right)$ and each of the images in the $T_{1}$ weighted series $\left(\mathrm{SI}_{\mathrm{T}_{1}}\right)$. These data were then used to calculate an enhancement factor (EF) time series proportional to the concentration of gadopentetate dimeglumine, with differences/changes in the native tissue $T_{1}$ between patients/visits being corrected for using the proton density SI and the mean pre-contrast $T_{1} \mathrm{SI}$ as obtained from a user-defined number of baseline points $\left(\mathrm{SI}_{0}\right)$ (Hittmair et al, 1994). The equations for $\mathrm{EF}$ calculation were $\mathrm{EF}(t)=1 /\left(K \mathrm{TR}_{\mathrm{T}_{1}}\right) \times \ln \left[\left(\mathrm{SI}_{\max }-\mathrm{SI}_{0}\right) /\left(\mathrm{SI}_{\max }-\mathrm{SI}_{\mathrm{T}_{1}}(t)\right)\right]$ and $\mathrm{SI}_{\max }=\mathrm{SI}_{\mathrm{PD}} \times \sin \left(\alpha_{\mathrm{T}_{1}} / \alpha_{\mathrm{PD}}\right)$, where $\alpha_{\mathrm{T}_{1}}$ and $\mathrm{TR}_{\mathrm{T}_{1}}$ are the flip angle and TR for the dynamic $T_{1}$-weighted series and $\alpha_{\mathrm{PD}}$ is the flip angle for the proton density-weighted image. A value of 11.04 was used for the constant $K$, this having been determined through calibration experiments using gels of known $T_{1}$ values and being valid for $\alpha_{\mathrm{T}_{1}}=30^{\circ}$ only.

A two-compartment PK model (Buckley et al, 1994; Tofts, 1997) was used to calculate the amplitude, or initial slope, of the EF time series (this being proportional to the microvessel transfer constant $K^{\text {trans }}$ (Tofts, 1997), itself proportional to the permeability surface area product) and the rate constant $\left(K_{\mathrm{ep}}\right)$. The model equation was

$$
\begin{aligned}
\mathrm{EF}(t)= & K^{\text {trans }} /\left(K_{\mathrm{ep}}-K_{\mathrm{el}}\right) \\
& {\left[\exp \left(-K_{\mathrm{el}}\left(t-t_{0}\right)\right)-\exp \left(-K_{\mathrm{ep}}\left(t-t_{0}\right)\right)\right], }
\end{aligned}
$$

where $K_{\mathrm{el}}$ represents contrast clearance from the plasma and $t_{0}$ represents the variable arrival time of the contrast agent. The extracellular-extravascular tissue volume fraction $\left(V_{\mathrm{e}}\right)$ was also estimated using the $K^{\text {trans }} / K_{\text {ep }}$ ratio (assuming equal influx and efflux vascular permeability; Tofts, 1997). Curve fitting was carried out (using mean ROI data, not pixel-by-pixel) using a nonlinear least-squares algorithm implemented within the in-house, IDL software, and the model-independent maximum enhancement factor (MEF) was also recorded.

Tumour volume was measured using manually traced ROIs drawn on high-resolution three-dimensional, post-contrast, fatsuppressed FSPGR images. Again, sequence acquisition parameters were varied in order to obtain adequate tissue coverage in all cases and these ranged as follows: TR/TE/flip $=14.6 / 4.2 \mathrm{~ms}$ fractional $/ 30^{\circ}$; plane $/ \mathrm{FOV} / \mathrm{matrix}=$ sagittal $/ 18-24 \mathrm{~cm} / 512 \times 256$ slice number/thickness $=25 / 2.5-5 \mathrm{~mm}$. Tumour volumes were measured, rather than more typical linear measurements, as this was considered to be the most rigorous and accurate, although most time-consuming, way of quantifying lesion size. Tumour volume has been shown to correlate more closely than tumour diameter with disease-free survival in breast cancer patients and it may also provide a more sensitive characterisation of tumour response than one-dimensional measurements (Partridge et al, 2005).

It was assumed that neoplastic tissue would not demonstrate substantial ADC anisotropy; therefore, in an attempt to keep scan time to a minimum, ADC was measured in the frequency-encode direction only (left-to-right), which was known to demonstrate the best imaging homogeneity and stability. The Stejskal-Tanner technique (Stejskal and Tanner, 1965) was implemented using a single fat suppressed slice (axial, $22 \mathrm{~cm}$ FOV, $128^{2}$ matrix, $7 \mathrm{~mm}$ thick) single-shot, spin-echo echo-planar imaging and with the 
following diffusion weightings: $0,14,55,125,222,346,499$ and $680 \mathrm{~s} \mathrm{~mm}^{-2}$. The ADC values were calculated on a whole ROI basis (Gibbs et al, 2001), which included a correction for the bias inherent in magnitude image reconstruction at low signal-to-noise ratios (especially true for higher $b$-values) (Miller and Joseph, 1993).

The relative proportions of spectroscopic signal arising from tissue water and lipids were measured at echo times of 30 and $135 \mathrm{~ms}$ using a one-dimensional stimulated echo (STEAM) spectroscopic imaging sequence (without chemical shift selective suppression of any moieties) and a repetition time of $3 \mathrm{~s}$. Thirtytwo phase-encode steps were used over a $16 \mathrm{~cm}$ superior-inferior FOV with STEAM excitation limited to seven adjacent voxels $(1.0 \times 0.5 \mathrm{~cm}$ in the anterior-posterior and left-right directions, respectively, giving a nominal voxel volume of $0.25 \mathrm{ml}$ ). Figure 1 shows the seven spectroscopic voxels in a representative tumour before chemotherapy. To ensure that the same region of tissue was examined in follow-up studies, MRS voxels were relocated, by an experienced radiologist, after careful comparison of the breast architecture surrounding the lesion with the architecture demonstrated on hard copies of previous voxel locations. Changes in tumour choline were not measured, as the predictive utility of the technique had not been established in the literature at the time of study design.

Spectral analysis was carried out using the SAGE IDL package (International General Electric, Milwaukee, WI, USA). In order to decrease the degree of inter-voxel signal contamination, mild spatial apodisation was applied (Fermi filter: diameter/ width $=90 \% / 5 \%$ ), which resulted in an effective voxel volume of $0.33 \mathrm{ml}$ (Jackson et al, 1994). To minimise partial volume contamination from adipose tissues outside the tumours, only signals from those voxels contained wholly within the tumour were averaged; then, water and fat signal intensities were measured by frequency-domain fitting of the water peak (4.7 p.p.m.) and the dominant lipid peaks at 1.3 and 0.9 p.p.m. (representing the

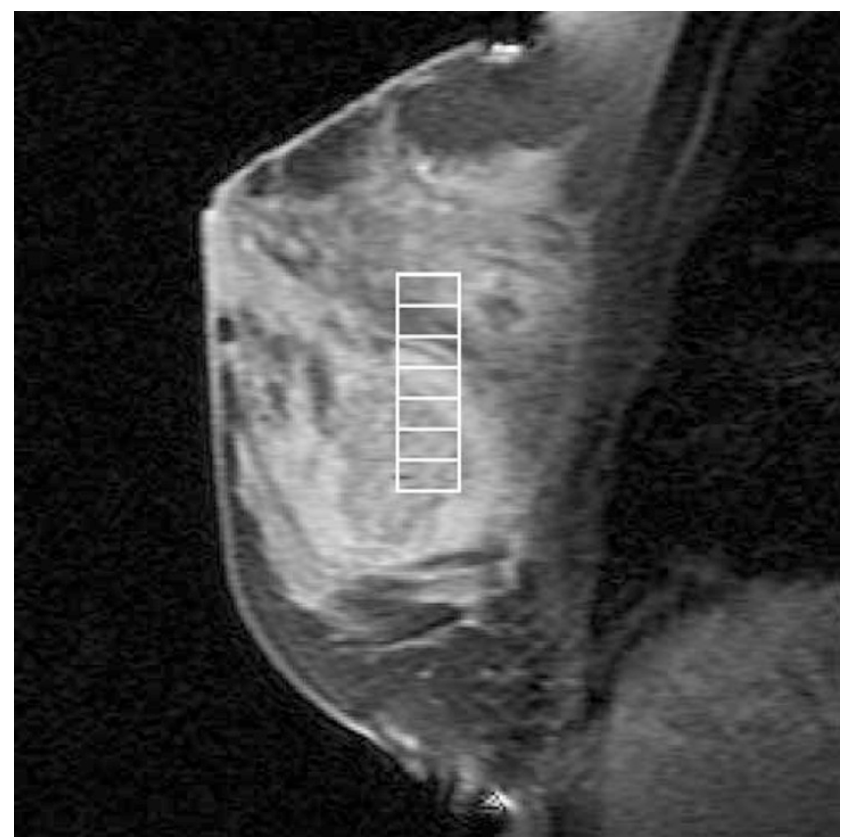

Figure I An example of the seven-voxel spectroscopic imaging localisation technique in a pre-chemotherapy tumour. (The bottom two voxels can be seen to contain wholly tumour, and spectral analysis would have been limited to these voxels.) The image on which the voxels are overlaid is the single slice, $T_{1}$-weighted, sagittal localiser scan acquired with fat suppression post-contrast and immediately before spectroscopy. methylene - $\left(\mathrm{CH}_{2}\right)_{n}$ - and terminal methyl $-\mathrm{CH}_{3}$ lipid moieties, respectively). Spectroscopic water content ( $\% W_{\text {MRS }}$ ) was then quantified using the ratio of the water signal to that of the sum of water and the two dominant fat signals. Water $T_{2}$ was also estimated using a crude two-point method: $T_{2} \sim\left(\mathrm{TE}_{135}-\mathrm{TE}_{30}\right) /$ $\ln \left(\operatorname{area}_{30} / \operatorname{area}_{135}\right)$.

\section{Biopsy data}

Patients' notes were reviewed to collate data obtained from histopathological analysis of specimens acquired via pre-chemotherapy core biopsy and final surgery. Parameters of interest were histological tumour subtype, tumour grade (pre-chemotherapy), the presence or absence of ductal carcinoma in situ (DCIS) and pre-chemotherapy oestrogen and progesterone receptor status (ERS and PRS) scores. Oestrogen and progesterone receptor status scores were recorded in the notes on either a six- or eight-point scale and converted to continuous data by calculating the ratio of the score to maximum possible score. The maximum diameter of the resected tumour, as calculated by the pathologist, was also collated and compared to the maximum diameter recorded in the final clinical MRI report using the Limits of Agreement method proposed by Bland and Altman (1986).

\section{Quantifying and correlating parameter changes and tumour response}

Changes in parameter values between $\mathrm{TP}_{0}$ and $\mathrm{TP}_{2}$ were calculated either as absolute differences, $D_{02}(X)=X_{\mathrm{TP}_{2}}-X_{\mathrm{TP}_{0}}$, or percentage change, $\mathrm{PC}_{02}(X)=X_{\mathrm{TP}_{2}} / X_{\mathrm{TP}_{0}}-1$, as deemed most appropriate. A change in tumour volume at the end of treatment, $\operatorname{PC}_{0 \mathrm{~F}}(V)$, exceeding $-65 \%$ was taken as the cutoff between partial response (PR) and stable disease (SD), this being equivalent to a decrease in cross-sectional area of $50 \%$, itself broadly equivalent to the RECIST criterion of a $50 \%$ decrease in the product of maximum orthogonal diameters (Therasse et al, 2000). No attempt was made to predict final tumour size in this study, as this is not likely to alter patient management before surgery.

All statistical analyses were carried out using the SPSS package (SPSS Inc., Chicago, IL, USA). Correlation between $D_{02}(X), \mathrm{PC}_{02}(X)$ or continuous/ranked pathology data and $\mathrm{PC}_{0 \mathrm{~F}}(V)$ was assessed using the Spearman non-parametric test (two-tailed). Correlation between dichotomous pathology data (presence/absence of DCIS) and categorical pathology data (cancer type) was assessed using the Mann-Whitney and Kruskal-Wallis non-parametric tests (two-tailed), respectively. Prognostic efficacy was assessed using receiver-operator characteristic (ROC) curves (Altman and Bland, 1994), with PR as the positive result, and the areas contained underneath them (AUC) calculated.

Variables were combined, in the hope of attaining a synergistic increase in prognostic efficacy, using logistic regression analysis (LRA) modelling, a statistical technique that maximises binary classification accuracy using a linear combination of weighted input variables plus a constant term (Hosmer and Lemeshow, 1989). Backwards conditional elimination of input variables $(P>0.10)$ was used in order to prevent over-parameterisation of models, and ordinal variables were treated as categorical with the most benign category used as the indicator reference. Some statistical confidence intervals (CIs) were obtained using tabulated values (Anonymous, 1982).

\section{RESULTS}

\section{Patient recruitment, withdrawal and other excluding factors}

A total of 46 women were recruited into the study over a period of approximately 2 years; however, six (13\%) of these subsequently 
withdrew. One case of atypical, inflammatory lobular cancer was excluded from data analysis on the grounds that it is impossible to measure tumour volume accurately in such tumours. One case in which histology only revealed DCIS was also excluded on the grounds that DCIS is too dissimilar to the other tumour types present, and chemotherapy is not the treatment of choice for it $(2 / 40=5 \%)$.

In three of the remaining 38 cases, the second MRI examination was severely delayed owing to clinical complications and was carried out after the chosen cutoff of 55 days, thus necessitating the exclusion of the $\mathrm{TP}_{2}$ (but not the $\mathrm{TP}_{0}$ ) data. In addition, it was not possible to acquire ADC and MRS data on every occasion because of a number of problems including hardware failure and an inability to acquire sufficient signal to permit sequence optimisation. Such failures occurred during the $\mathrm{TP}_{0}$ scan, necessitating complete exclusion of the patient from data analysis, in four cases, and at $\mathrm{TP}_{2}$, necessitating removal of $\mathrm{TP}_{2}$ data only, in nine cases. A total of 13 failures in the 73 examinations ( 38 at $\mathrm{TP}_{0}$ and 35 at $\mathrm{TP}_{2}$ ) represent a failure rate of $18 \%$. Results will, therefore, be presented for 22 cases for which both $\mathrm{TP}_{0}$ and $\mathrm{TP}_{2}$ data were available and an additional 12 cases for which data were available at $\mathrm{TP}_{0}$ only (see Figure 2).

Neither tumour volume measurements nor PK analysis were affected at any time point, as these were based on threedimensional and DCE-MRI images acquired before ADC and MRS were attempted. Therefore, tumour volume response, $\mathrm{PC}_{\mathrm{OF}}(V)$, could be quantified in all cases. Pharmacokinetic data and tumour volume data calculated in cases where ADC and MRS data were not acquired were voluntarily excluded, so as not to bias statistical power in favour of PK/volume parameters. In the 22 cases for which valid $\mathrm{TP}_{2}$ data were available, $18(82 \%)$ of the women had received two courses of chemotherapy at the time of MRI and four had received three courses. In three cases, it was decided to cease chemotherapy before a full six cycles were completed, as poor clinical response was being demonstrated. In these cases, MRI was, however, carried out after the final course of treatment, thus permitting a final measurement of tumour volume to be made and, thus, quantifying the relatively poor response adequately.

All five pathology variables (i.e. cancer type, grade, presence/ absence of DCIS, and ERS and PRS scores) were available in 35 of the 38 eligible cases. These included 32 of the 34 valid $\mathrm{TP}_{0} \mathrm{MRI}$ cases, all 22 of the valid $\mathrm{TP}_{2}$ MRI cases and three cases in which MRI data were unusable (see Figure 2). The age at $\mathrm{TP}_{0}$ for the 37 women whose data are used in this study ranged from 26 years, 6 months to 75 years, 7 months, with a median of 48 years, 6 months.

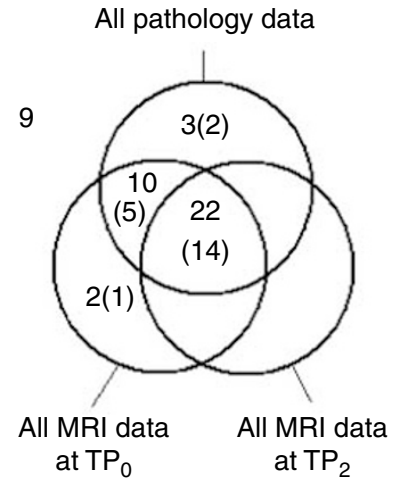

Figure 2 A Venn diagram showing the availability of valid data for the 46 women recruited into this study (with nine cases having to be excluded completely). Numbers in parentheses indicate the number of cases where DCIS was present.
The pathological cancer subtypes of these 37 cases were 19 ductal/ not specified (12 with DCIS), 15 (nine) ductal and three (one) lobular. The number of women with cancers of grade one, two and three were nine, 18 and eight, respectively (cancer grade was not reported by the pathologist in two cases). Two women did not undergo surgery because of the presence of metastatic disease and one woman did not undergo surgery as post-chemotherapy core biopsy confirmed the absence of residual cancer. In the remaining cases, 17 women underwent mastectomy and 17 underwent wide local excision. The length of time between the final MRI scan and surgery ranged from 14 to 117 days, with a median of 28 days. Maximum tumour diameter was recorded in the patient's notes by both the pathologist and the MRI radiologist in 21 cases.

\section{Agreement between MRI and pathology measurements}

Calculation of the limits of agreement between the maximum tumour diameters as measured by the pathologists and the MRI radiologist demonstrated a mean difference (MRI-pathology) of $-2.1 \mathrm{~mm}$ with a $95 \% \mathrm{CI}(1.96 \times$ standard deviation $)$ of $\pm 18.6 \mathrm{~mm}$. The limits of agreement were, therefore, -20.7 to $16.5 \mathrm{~mm}$. A paired $t$-test of the two sets of measurements showed that the mean difference was not statistically significant $(P=0.32)$, thus indicating that MRI is an unbiased estimator of true tumour size and, therefore, suitable for quantifying tumour response. The maximum diameter as measured by the MRI radiologist was also compared to the cube root of the final tumour volume, and these two variables demonstrated excellent linear correlation $\left(P=0.0001 ; R^{2}=0.56\right)$.

\section{Predicting response using individual variables}

The ranges of all predictive MRI variables are presented in Table 1, and the results of correlating the predictive MRI and pathology variables with $\mathrm{PC}_{0 \mathrm{~F}}(V)$ are given in Table 2 . Six MRI variables demonstrated $P$-values less than 0.07 and the data for these, divided into SD and PR subsets, are presented in Figure 3. These six variables along with four histopathology variables for which the $P$-values were also less than 0.07 underwent ROC curve analysis, and the results of this are also given in Table 2. The slight differences in the patterns of statistical significance between the correlation and ROC analyses can be attributed to the different variables quantifying response in the two types of tests (specifically that $\mathrm{PC}_{0 \mathrm{~F}}(V)$ is continuous whereas $\mathrm{PR} / \mathrm{SD}$ is dichotomous). Four variables demonstrated an ROC AUC significantly larger than 0.5: water $T_{2}$ at $\mathrm{TP}_{0}$ and changes in $\% W_{\mathrm{MRS}}$ measured at $135 \mathrm{~ms}$, water $T_{2}$ and tumour volume between $\mathrm{TP}_{0}$ and $\mathrm{TP}_{2}$. The ROC plots for these are shown in Figure 4.

Table I The range of values for all predictive MRI variables used in this study, as grouped by clinical time point ( $T P_{0}$ being before treatment and $\mathrm{TP}_{2}$ being after the second course of chemotherapy)

\begin{tabular}{|c|c|c|}
\hline Parameter (units) & $\mathrm{TP}_{0}(n=34)$ & $\mathrm{TP}_{2}(n=22)$ \\
\hline Volume (ml) & $3.7-240.0$ & $1.3-95.0$ \\
\hline$K^{\text {trans }}$ (per unit time) & $1.4-18.0$ & $0.6-8.3$ \\
\hline$K_{\mathrm{ep}}$ (per unit time) & $0.4-12.4$ & $0.4-3.9$ \\
\hline$V_{e}$ (arbitrary) & $0.6-3.5$ & $1.2-3.1$ \\
\hline MEF (arbitrary) & $0.8-3.5$ & $1.3-3.6$ \\
\hline $\operatorname{ADC}\left(\mathrm{mm}^{2} / \mathrm{s}\right)$ & $0.3-5.9$ & $0.9-4.3$ \\
\hline$\% W_{\text {MRS }}$ at $135 \mathrm{~ms}$ & $68-100 \%$ & $30-100 \%$ \\
\hline$\% W_{M R S}$ at $30 \mathrm{~ms}$ & $73-100 \%$ & $49-100 \%$ \\
\hline Water $T_{2}$ (ms) & $47-130$ & $24-104$ \\
\hline
\end{tabular}

$\mathrm{ADC}=$ apparent diffusion coefficient; $\mathrm{MEF}=$ maximum enhancement factor $\mathrm{MRI}=$ magnetic resonance imaging. 
Table 2 Statistical significance of non-parametric correlation analyses between the variables indicated and final tumour volume response $\mathrm{PC}_{\mathrm{OF}}(\mathrm{V})$

\begin{tabular}{|c|c|c|c|c|c|}
\hline & $\begin{array}{c}\text { No. of cases } \\
\text { PR+SD }\end{array}$ & $\begin{array}{l}\text { Correlation } \\
P \text { (sense) }\end{array}$ & \multicolumn{3}{|c|}{ ROC curve } \\
\hline $\begin{array}{l}\text { MRI data at } T_{2} \\
\mathrm{PC}_{02}(\text { tumour volume })^{[2]} \\
D_{02}\left(\text { water } T_{2}\right)^{[3]} \\
\mathrm{PC}_{02}\left(\% W_{\text {MRS }} \text { at } 135 \mathrm{~ms}\right)^{[4]} \\
D_{02}\left(K^{\text {trans }}\right) \\
D_{02}\left(V_{\mathrm{e}}\right) \\
D_{02}\left(K_{\text {ep }}\right) \\
D_{02}(\text { MEF }) \\
D_{02}(\text { water ADC }) \\
P_{02}\left(\% W_{\text {MRs }} \text { at } 30 \mathrm{~ms}\right)\end{array}$ & $\begin{array}{l}16+6 \\
16+6 \\
16+6 \\
16+6 \\
16+6 \\
16+6 \\
16+6 \\
16+6 \\
16+6\end{array}$ & $\begin{array}{l}\text { S: } 0.001(+) \\
\text { S: } 0.006(+) \\
\text { S: } 0.025(+) \\
\text { S: } 0.299 \\
\text { S: } 0.352 \\
\text { S: } 0.405 \\
\text { S: } 0.587 \\
\text { S: } 0.736 \\
\text { S: } 0.788\end{array}$ & $\begin{array}{l}0.03 \\
0.04 \\
0.02\end{array}$ & $\begin{array}{l}0.80 \\
0.79 \\
0.83\end{array}$ & $\begin{array}{l}0.58 \text { to } 1.00 \\
0.60 \text { to } 0.98 \\
0.60 \text { to } 1.00\end{array}$ \\
\hline $\begin{array}{l}\text { Other data } \\
\text { Age at TP } \\
\text { Cancer grade } \\
\text { Presence of DCIS } \\
\text { PRS score }{ }^{[6]} \\
\text { ERS score } \\
\text { Cancer type }\end{array}$ & $\begin{array}{l}26+11 \\
24+11 \\
24+11 \\
24+11 \\
24+11 \\
24+11\end{array}$ & $\begin{array}{l}\text { S: } 0.958 \\
\text { S: } 0.003(-) \\
\text { M: } 0.006(+) \\
\text { S: } 0.008(+) \\
\text { S: } 0.061(+) \\
\text { K: } 0.740\end{array}$ & $\begin{array}{l}0.08 \\
0.14 \\
0.09 \\
0.17\end{array}$ & $\begin{array}{l}0.69 \\
0.66 \\
0.68 \\
0.65\end{array}$ & $\begin{array}{l}0.49 \text { to } 0.88 \\
0.49 \text { to } 0.88\end{array}$ \\
\hline $\begin{array}{l}\text { LRA models (variables included) } \\
\text { MRI only } y^{[l, 2,4]} \\
\left.\text { MRI and histopathology }{ }^{[1} \text { to } 6\right]\end{array}$ & $\begin{array}{l}16+6 \\
16+6\end{array}$ & & $\begin{array}{l}0.0032 \\
0.0004\end{array}$ & $\begin{array}{l}0.92 \\
1.00\end{array}$ & $\begin{array}{l}0.79 \text { to } 1.00 \\
1.00 \text { to } 1.00\end{array}$ \\
\hline
\end{tabular}

The sense of the correlations is also shown. Results of ROC curve analyses (with PR/SD as positive/negative results) are also shown for selected variables. Numbers in square brackets indicate those variables chosen as inputs to LRA modelling. $\mathrm{S}=$ Spearman rank correlation; $\mathrm{M}=\mathrm{Mann}-\mathrm{Whitney}$ test; $\mathrm{K}=$ Kruskal - Wallis test; $(+)$ a positive correlation with lower, or more negative values being associated with more negative values of $\mathrm{PC}_{\mathrm{OF}}(\mathrm{V})$, that is, $\mathrm{PR} ;(-)$ a negative correlation with higher, or more positive values being associated with more negative values of $\mathrm{PC}_{\mathrm{OF}}(V)$, that is, $\mathrm{PR}$. $A \cup C=$ area under the curve; $A D C=$ apparent diffusion coefficient; $D C I S=$ ductal carcinoma in situ; $E R S=$ oestrogen receptor status; $L R A=$ logistic regression analysis; $M E F=$ maximum enhancement factor; $M R I=$ magnetic resonance imaging; $P R=$ partial response; $\mathrm{PRS}=$ progesterone receptor status; $\mathrm{ROC}=$ receiver - operator characteristic; $\mathrm{SD}=$ stable disease.
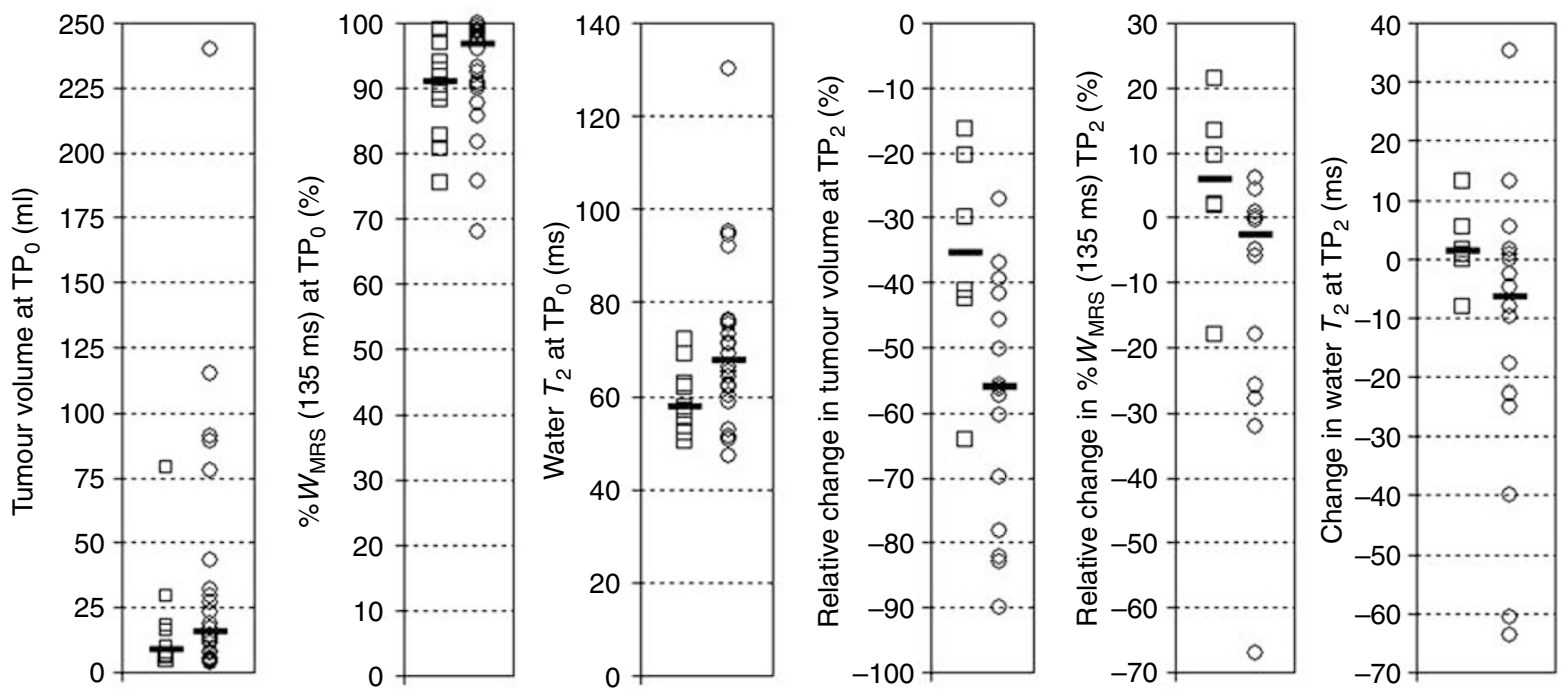

$\square$ Stable disease (SD) o Partial response (PR) - Medians

Figure 3 Dot plots showing the PR and SD data for six MRI variables that when correlated with a final change in tumour volume demonstrated $P$-values less than 0.07. The medians for both SD and PR subsets are also shown. 
When specificity is set at $100 \%$ in Figure 4 , the sensitivity for the change in water $T_{2}$ at $\mathrm{TP}_{2}$ is $69 \%(11 / 16 ; 95 \% \mathrm{CI}=41-89 \%)$, which suggests that this fraction of PR cases can be accurately predicted, that is, at $100 \%$ positive predictive value (PPV). Similarly, when sensitivity is set to $100 \%$, the specificity displayed by the change in $\% W_{\mathrm{MRS}}$ at $\mathrm{TP}_{2}$ is $50 \%(3 / 6 ; 95 \% \mathrm{CI}=12-88 \%)$, which suggests that this fraction of SD cases can be accurately predicted, that is, at $100 \%$ negative predictive value (NPV) by this variable. The results of Table 2 also suggest that the dominant factor in driving the observed changes in \% $W_{\text {MRS }}$ at $135 \mathrm{~ms}(P=0.025)$ is the change in water $T_{2}(P=0.006)$ as opposed to changes in the relative water and lipid concentrations, which would be reflected by changes in $\% W_{\text {MRS }}$ at $30 \mathrm{~ms}(P=0.788)$ for which the effects of $T_{2}$ would be much less.

\section{Predicting response using a combination of variables}

All variables demonstrating an ROC $P$-value less than 0.10 (Table 2) were presented as inputs to LRA modelling. Two separate analyses were conducted: one with MRI variables only (water $T_{2}$ at $\mathrm{TP}_{0}, \mathrm{PC}_{20}$ (tumour volume), $D_{20}$ (water $T_{2}$ ) and $\mathrm{PC}_{20}\left(\% W_{\mathrm{MRS}}\right.$ at $\left.\left.135 \mathrm{~ms}\right)\right)$ and one with both MRI and pathology data (PRS score and grade). The number of cases that could be included in LRA modelling was limited to those 22 for which all of the above variables were available (16 PR and six SD). The logits for the two final models were as follows:

$$
\begin{aligned}
\text { Logit } 1= & 114.9 \times\left[\mathrm{T}_{2} \text { at } \mathrm{TP}_{0}\right]-10.1 \times \mathrm{PC}_{20}(\text { tumour volume }) \\
& -10.9 \times \mathrm{PC}_{20}(\% W \text { at } 135 \mathrm{~ms})-11.1
\end{aligned}
$$

$$
\begin{aligned}
\text { Logit } 2= & 19709.5 \times\left[\mathrm{T}_{2} \text { at } \mathrm{TP}_{0}\right] \\
& -1890.1 \times \mathrm{PC}_{20}(\text { tumour volume }) \\
& -1877.9 \times \mathrm{PC}_{20}(\% W \text { at } 135 \mathrm{~ms}) \\
& +6330.0 \times D_{20}\left(\text { water } T_{2}\right) \\
& -785.6 \times[\text { PRS score }]-310.4[\text { IF grade } 1] \\
& -111.0[\text { IF grade } 3]-1245.6
\end{aligned}
$$

The ROC AUC values for the above logits are shown in Table 2. Both models provide an increase in prognostic accuracy over all of the individual variables, and the second model is able to classify all 22 cases accurately. Both PPV and NPV are 100\% for this data set and the $95 \%$ CI lower bounds are $79 \%(16 / 16)$ and $54 \%(6 / 6)$, respectively.

\section{DISCUSSION}

To the authors' knowledge, this paper represents the first study whereby PK parameter mapping, ADC mapping and unsuppressed proton spectroscopy (water: fat ratios) have all been investigated in the same patient examinations, thus permitting their relative abilities to predict chemotherapy response to be ascertained rigorously. The use of spectroscopic imaging at both short and long echo times represents a significant extension to the methodology of Jagannathan et al (1998): firstly through permitting greater post-examination control over intra-voxel partial volume effects and secondly by permitting the effects of $T_{2}$ relaxation to be revealed. It was also hoped that the $30 \mathrm{~ms} \mathrm{TE}$ spectra would permit investigation of the relative proportions of the 1.3 and 0.9 p.p.m. lipid peaks, along with the contributions of other, minor lipid moieties, which are more conspicuous at short TE. This analysis is not possible with quantitative chemical shift imaging (Daniel et al, 1998), as this technique, while having much higher spatial resolution, only provides images for water and total fat signal. It did not prove possible to resolve the separate lipid components adequately in a sufficient number of cases to permit this; therefore, chemical shift imaging may be a suitable alternative

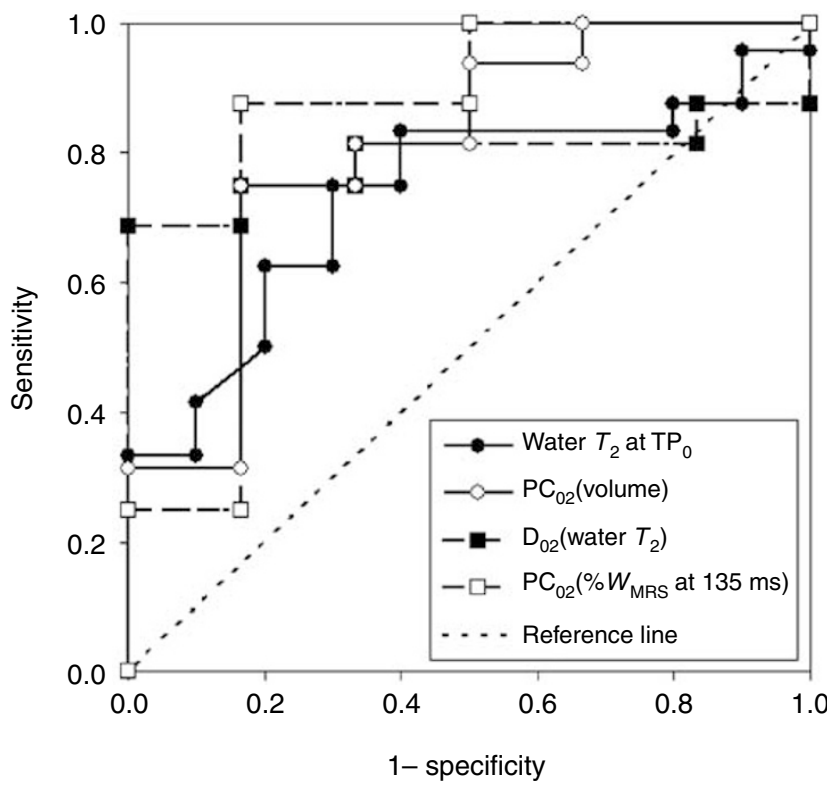

Figure 4 Receiver-operator characteristic curves, with PR as the positive result, for the baseline ( $\left.T P_{0}\right)$ water $T_{2}$ (24 PR and I0 SD data) and the changes between $\mathrm{TP}_{0}$ and $\mathrm{TP}_{2}$ in spectroscopic water content measured at $135 \mathrm{~ms}$, tumour volume and water $T_{2}$ ( I 6 PR and six SD data) Diagonal segments are caused by ties.

to spectroscopic imaging, permitting increased spatial resolution in future studies.

This study shows that MRS parameters provide substantial prognostic information, and slightly more than that provided by volume measurements alone, thus supporting the conclusions of Jagannathan et al (1998). This study also suggests that water $T_{2}$ plays a dominant part in driving the observed treatment changes in water: fat ratios $\left(\right.$ at $\mathrm{TP}_{2}$ ) and it is, therefore, possible that imagebased $T_{2}$ mapping techniques (Liney et al, 1996) might provide the same prognostic information as $\% W_{\text {MRS }}$ but with the combined benefits of greatly increased spatial resolution, wider availability in clinical MRI centres and a reduced likelihood of technical failure in a given examination. Quantitative $T_{2}$ mapping has been applied to monitoring chemotherapy in one animal tumour study, but the increases in $T_{2}$ observed 3 days post-therapy were not statistically significant (Duvvuri et al, 2001). Significant differences were observed for $T_{1 \rho}$, however - a parameter not measured in this study because of time and technical constraints. Whether chemotherapy induces cell death via necrosis, apoptosis or a combination of the two, profound changes will certainly occur in the molecular environment of tissue water, leading to changes in its measured $T_{2}$. This molecular environment is known to be complex and multicompartmental, which precludes a simple rationale for the results of this study.

Although water:fat ratios have been shown to contain substantial prognostic power, in both this and another study (Jagannathan et al, 1998), it is noted that a number of groups are now focusing on measurements of choline-containing compounds using water-suppressed MRS, as the choline moiety is potentially a more sensitive, and probably a more biochemically relevant marker, of cancer cell viability. Such studies have recently been carried out in breast cancer (Jagannathan et al, 2001; Meisamy et al, 2004), lymphoma and germ cell tumours (Schwarz et al, 2002), and glioma (Preul et al, 2000), where positive treatment response has been associated with reduction in the levels of total choline. Similar studies using phosphorous spectroscopy to detect changes in phosphocholine or phosphomonoesters have also been 
carried out in lymphoma (Arias-Mendoza et al, 2004) and head and neck cancer (Shukla-Dave et al, 2002a, b). It is noteworthy that these studies attempted to predict response before treatment commencement, always a more valuable prognostic test, sparing the patients all unnecessary morbidity and time, than reliably detecting early response, which the methods presented herein achieve.

This study also demonstrates that histopathology data routinely available from pre-chemotherapy core biopsies also provide substantial prognostic power (especially so for PRS score and grade). This conclusion is supported by other laboratory studies, which have included investigation of expression of the HER2 oncogene (Penault-Llorca et al, 2003) and the cellular proliferation marker Ki-67 (Aas et al, 2003). Whereas laboratory analysis of actual tissue is inherently superior to imaging alone, it should be noted that the core biopsy technique is always prone to sampling errors, whereas imaging has the advantage of covering the whole of the lesion.

This study also demonstrates that a combination of prognostic variables, via LRA, can be used to provide a synergistic increase in prognostic accuracy, thus proving the study hypothesis. Although the perfect prognostic accuracy achieved using one of the LRA models indicates that the methods used in this study have substantial prognostic power and could be applied clinically, leading to benefits for patients, the true accuracy will be less and will need to be ascertained in larger studies. A multicentre study would also allow investigation of reproducibility. It might also be beneficial to split the data acquired in subsequent studies into separate test and validation subsets, thus allowing optimal model coefficients to be set with the former subset and their true prognostic accuracy determined through application of the model to the completely independent data in the latter.

Early, accurate detection of non-response to chemotherapy would permit an early change to second-line treatment and thus spare patients unnecessary toxicity, cost and delay of initiation of effective treatment. The cessation of treatment that would ultimately prove ineffective might also be beneficial in terms of health economics, allowing resources to be applied more efficiently. Conversely, early, accurate detection of response to chemotherapy might have positive benefits for the psychological well-being and quality-of-life of patients through giving them increased hope. The high prognostic accuracy might be bolstered by including variables that quantify lesion shape and texture, as obtained from the post-contrast three-dimensional images, in the LRA modelling (Esserman et al, 2001).

This study shows that ADC mapping after the second course of chemotherapy does not contribute significantly towards detecting early response. Other studies have shown that treatment-induced changes in ADC in human breast cancer and breast cancer metastasised to liver are most marked a few days after the first dose of chemotherapy (Theilmann et al, 2003). Therefore, our study protocol, with the predictive scans being carried out after two cycles of chemotherapy rather than one (Gibbs et al, 2003), was perhaps not well suited to elucidating the relative merits of ADC mapping.

In contrast to our results, other studies have indicated that $\mathrm{PK}$ parameters derived from DCE-MRI contain prognostic information during chemotherapy treatment in a number of locations in the human body (with reductions in $K^{\text {trans }}$ being associated with positive response) as reviewed by Padhani (2002) and Knopp et al (2003). This apparent discrepancy regarding the efficacy of PK parameters may be due to the relatively small number of cases included in various studies, or the wide variety of DCE-MRI acquisition and PK analysis techniques used. Intra-tumour vascular heterogeneity might also be significant in some breast cancers (Hayes et al, 2002), leading to the possible breakdown of PK model assumptions such as the fast exchange limit within better-perfused regions (usually located at the tumour rim) (Zhou et al, 2004; Li et al, 2005). This could present a complicating factor in obtaining reliable results, an issue not addressed in this study, which utilised a whole ROI approach so as to minimise the effects of intra-scan patient motion.

Some DCE-MRI studies of breast cancer have attempted to improve the accuracy of PK modelling by imaging in the axial plane so as to allow a plasma concentration time-course to be measured in the aorta, thus permitting the calculation of an arterial input function (AIF) for the tumour. It is debatable, however, whether the benefits of including an AIF measured so distantly from the tissue of interest (i.e. one which does not allow for bolus dispersion effects) outweigh the reduction in spatial resolution and tissue coverage necessitated by axial imaging, especially when the DCE-MRI images are also used for clinical purposes. A consensus on the adoption of standardised techniques and the establishment of large, multicentre studies would, therefore, seem appropriate.

\section{ACKNOWLEDGEMENTS}

We gratefully acknowledge the financial support of Yorkshire Cancer Research and also Catherine Bowker, Lynn Cawkwell, John Greenman, John Read and Irvine Long for assistance with study design, patient recruitment, data acquisition and data analysis.

\section{REFERENCES}

Aas T, Geisler S, Eide GE, Haugen DF, Varhaug JE, Bassoe AM, Thorsen T, Berntsen H, Borresen-Dale AL, Akslen LA, Lonning PE (2003) Predictive value of tumour cell proliferation in locally advanced breast cancer treated with neoadjuvant chemotherapy. Eur J Cancer 39: 438 - 446

Altman DG, Bland JM (1994) Diagnostic tests 3 - receiver operating characteristic plots. BMJ 309: 188

Anonymous (1982) Geigy Scientific Tables. Basle: Geigy

Arias-Mendoza F, Smith MR, Brown TR (2004) Predicting treatment response in non-Hodgkin's lymphoma from the pretreatment tumor content of phosphoethanolamine plus phosphocholine. Acad Radiol 11: 368-376

Bland JM, Altman DG (1986) Statistical methods for assessing agreement between two methods of clinical measurement. Lancet 1: 307-310

Bolan PJ, Meisamy S, Baker EH, Lin J, Emory T, Nelson M, Everson LI, Yee $\mathrm{D}$, Garwood M (2003) In vivo quantification of choline compounds in the breast with H-1 MR spectroscopy. Magn Reson Med 50: 1134-1143
Bradamante S, Barchiesi E, Pilotti S, Borasi G (1988) High-resolution H-1NMR spectroscopy in the diagnosis of breast-cancer. Magn Reson Med 8: $440-449$

Buckley DL, Kerslake RW, Blackband SJ, Horsman A (1994) Quantitative analysis of multisclice GD-DTPA enhanced dynamic MR-images using an automated simplex minimisation procedure. Magn Reson Med 32: $646-651$

Buscombe JR, Cwikla JB, Thakrar DS, Hilson AJW (1997) Scintigraphic imaging of breast cancer: a review. Nucl Med Commun 18: 698-709

Byrne AM, Hill ADK, Skehan SJ, McDermott EW, O'Higgins NJ (2004) Positron emission tomography in the staging and management of breast cancer. Br J Surg 91: 1398-1409

Chu DZJ, Yamanashi WS, Frazer J, Hazlewood CF, Gallager HS, Boddie AW, Martin RG (1987) Proton NMR of human breast tumors correlation with clinical prognostic parameters. J Surg Oncol 36: 1-4 
Daniel BL, Butts K, Glover GH, Cooper C, Herfkens RJ (1998) Breast cancer: gadolinium-enhanced MR imaging with a $0.5 \mathrm{~T}$ open imager and threepoint Dixon technique. Radiology 207: 183-190

Drew PJ, Kerin MJ, Mahapatra T, Malone C, Monson JRT, Turnbull LW, Fox JN (2001) Evaluation of response to neoadjuvant chemoradiotherapy for locally advanced breast cancer with dynamic contrast-enhanced MRI of the breast. Eur J Surg Oncol 27: 617-620

Duvvuri U, Poptani H, Feldman M, Nadal-Desbarats L, Gee MS, Lee WMF, Reddy R, Leigh JS, Glickson JD (2001) Quantitative T1-rho magnetic resonance imaging of RIF-1 tumors in vivo: detection of early response to cyclophosphamide therapy. Cancer Res 61: 7747-7753

Esserman L, Hylton N, Yassa L, Barclay J, Frankel S, Sickles E (1999) Utility of magnetic resonance imaging in the management of breast cancer: evidence for improved preoperative staging. J Clin Oncol 17: 110-119

Esserman L, Kaplan E, Partridge S, Tripathy D, Rugo H, Park J, Hwang S, Kuerer H, Sudilovsky D, Lu Y, Hylton N (2001) MRI phenotype is associated with response to doxorubicin and cyclophosphamide neoadjuvant chemotherapy in stage III breast cancer. Ann Surg Oncol 8: $549-559$

Gallons JP, Altbach G, Paine-Murrieta C, Taylor C, Gillies RJ (1999) Early increases in breast tumor xenograft water mobility in response to paclitaxel therapy detected by non-invasive diffusion magnetic resonance imaging. Neoplasia 1: 113-117

Gibbs JE, Partridge SC, Hylton NM (2003) Incremental value of ADC as an indicator of treatment response in patients undergoing neoadjuvant chemotherapy for locally advanced breast cancer. Proc Int Soc Magn Reson Med 11: 291

Gibbs P, Tozer DJ, Liney GP, Turnbull LW (2001) Comparison of quantitative T2 mapping and diffusion-weighted imaging in the normal and pathologic prostate. Magn Reson Med 46: 1054-1058

Hayes C, Padhani AR, Leach MO (2002) Assessing changes in tumour vascular function using dynamic contrast-enhanced magnetic resonance imaging. NMR Biomed 15: 154-163

Hittmair K, Gomiscek G, Langenberger K, Recht M, Imhof H, Kramer J (1994) Method for the quantitative assessment of contrast agent uptake in dynamic contrast-enhanced MRI. Magn Reson Med 31: 567-571

Hosmer DW, Lemeshow S (1989) Applied Logistic Regression. Chichester: Wiley

Jackson EF, Doyle TJ, Wolinsky JS, Narayana PA (1994) Short TE H-1 spectroscopic MR-imaging of normal human brain - reproducibility studies. JMRI J Magn Reson Imaging 4: 545 - 551

Jacobs MA, Barker PB, Argani P, Ouwerkerk R, Bhujwalla ZM, Bluemke DA (2005) Combined dynamic contrast enhanced breast MR and proton spectroscopic imaging: a feasibility study. J Magn Reson Imaging 21: $23-28$

Jacobs MA, Barker PB, Bottomley PA, Bhujwalla Z, Bluemke DA (2004) Proton magnetic resonance spectroscopic imaging of human breast cancer: a preliminary study. J Magn Reson Imaging 19: 68 - 75

Jagannathan NR, Kumar M, Seenu V, Coshic O, Dwivedi SN, Julka PK, Srivastava A, Rath GK (2001) Evaluation of total choline from in vivo volume localized proton MR spectroscopy and its response to neoadjuvant chemotherapy in locally advanced breast cancer. $\mathrm{Br} J$ Cancer 84: 1016 - 1022

Jagannathan NR, Singh M, Govindaraju V, Raghunathan P, Coshic O, Julka PK, Rath GK (1998) Volume localized in vivo proton MR spectroscopy of breast carcinoma: variation of water-fat ratio in patients receiving chemotherapy. NMR Biomed 11: 414-422

Katz-Brull R, Lavin PT, Lenkinski RE (2002) Clinical utility of proton magnetic resonance spectroscopy in characterizing breast lesions. J Nat Cancer Inst 94: 1197 - 1203

Kline RP, Wu EX, Petrylak DP, Szabolcs M, Alderson PO, Weisfeldt ML, Cannon P, Katz J (2000) Rapid in vivo monitoring of chemotherapeutic response using weighted sodium magnetic resonance imaging. Clin Cancer Res 6: 2146 - 2156

Knopp MV, von Tengg-Kobligk H, Choyke PL (2003) Functional magnetic resonance imaging in oncology for diagnosis and therapy monitoring. Mol Cancer Ther 2: 419-426

Kumar R, Alavi A (2004) Fluorodeoxyglucose-PET in the management of breast cancer. Radiol Clin N Am 42: 1113-1122

Li X, Huang W, Yankeelov TE, Tudorica A, Rooney WD, Springer CS (2005) Shutter-speed analysis of contrast reagent bolus-tracking data: preliminary observations in benign and malignant breast disease. Magn Reson Med 53: 724-729

Liney GP, Knowles AJ, Manton DJ, Turnbull LW, Blackband SJ, Horsman A (1996) Comparison of conventional single echo and multi-echo sequences with a fast spin echo sequence for quantitative T2 mapping: application to the prostate. JMRI J Magn Reson Imaging 6: $603-607$

Meisamy S, Bolan PJ, Baker EH, Bliss RL, Gulbahce E, Everson LI, Nelson MT, Emory TH, Tuttle TM, Yee D, Garwood M (2004) Neoadjuvant chemotherapy of locally advanced breast cancer: predicting response with in vivo H-1 MR spectroscopy - a pilot study. Radiology 233: 424-431

Miller AJ, Joseph PM (1993) The use of power images to perform quantitative analysis on low SNR MR images. Magn Reson Imaging 11: $1051-1056$

Moffat BA, Chenevert TL, Lawrence TS, Meyer CR, Johnson TD, Dong Q, Tsien C, Mukherji S, Quint DJ, Gebarski SS, Robertson PL, Junck LR, Rehemtulla A, Ross BD (2005) Functional diffusion map: a noninvasive MRI biomarker for early stratification of clinical brain tumor response. Proc Natl Acad Sci USA 102: 5524-5529

Padhani AR (2002) Dynamic contrast-enhanced MRI in clinical oncology: current status and future directions. J Magn Reson Imaging 16: 407-422

Partridge SC, Gibbs JE, Lu Y, Esserman LJ, Tripathy D, Wolverton DS, Rugo HS, Hwang GE, Ewing CA, Hylton NM (2005) MRI measurements of breast tumor volume predict response to neoadjuvant chemotherapy and recurrence-free survival. Am J Roentgenol 184: 1774-1781

Penault-Llorca F, Cayre A, Mishellany FB, Amat S, Feillel V, Le Bouedec G, Ferriere JP, De Latour M, Chollet P (2003) Induction chemotherapy for breast carcinoma: predictive markers and relation with outcome. Int Oncol 22: $1319-1325$

Preul MC, Caramanos Z, Villemure JG, Shenouda G, LeBlanc R, Langleben A, Arnold DL (2000) Using proton magnetic resonance spectroscopic imaging to predict in vivo the response of recurrent malignant gliomas to tamoxifen chemotherapy. Neurosurgery 46: 306-318

Ross BD, Moffat BA, Lawrence TS, Mukherji SK, Gebarski SS, Quint DJ, Johnson TD, Junck L, Robertson PL, Muraszko KM, Dong Q, Meyer CR, Bland PH, McConville P, Geng HR, Rehemtulla A, Chenevert TL (2003) Evaluation of cancer therapy using diffusion magnetic resonance imaging. Mol Cancer Ther 2: 581-587

Schepkin VD, Ross BD, Chenevert TL, Rehemtulla A, Sharma S, Kumar M, Stojanovska J (2005) Sodium magnetic resonance imaging of chemotherapeutic response in a rat glioma. Magn Reson Med 53: 85-92

Schwarz AJ, Maisey NR, Collins DJ, Cunningham D, Huddart R, Leach MO (2002) Early in vivo detection of metabolic response: a pilot study of $\mathrm{H}-1$ MR spectroscopy in extracranial lymphoma and germ cell tumours. $\mathrm{Br} J$ Radiol 75: 959-966

Shukla-Dave A, Poptani H, Loevner LA, Mancuso A, Serrai H, Rosenthal DI, Kilger AM, Nelson DS, Zakian KL, Arias-Mendoza F, Rijpkema M, Koutcher JA, Brown TR, Heerschap A, Glickson JD (2002a) Prediction of treatment response of head and neck cancers with P-31 MR spectroscopy from pretreatment relative phosphomonoester levels. Acad Radiol 9: $688-694$

Shukla-Dave A, Poptani H, Loevner LA, Mancuso A, Serrai H, Rosenthal DI, Kilger AM, Nelson DS, Zakian KL, Arias-Mendoza F, Rijpkema M, Koutcher JA, Brown TR, Heerschap A, Glickson JD (2002b) Prediction of treatment response of head and neck cancers with P-31 MR spectroscopy from pretreatment relative phosphomonoester levels (vol 9, pg 688, 2002). Acad Radiol 9: 772

Sijens PE, Wijrdeman HK, Moerland MA, Bakker CJG, Vermeulen JWAH, Luyten PR (1988) Human breast cancer in vivo - H-1 and P-31 MR spectroscopy at 1.5 T. Radiology 169: 615-620

Stejskal EO, Tanner JE (1965) Spin diffusion measurements: spin echoes in the presence of a time dependent field gradient. J Chem Phys 42: $288-292$

Theilmann RJ, Xia G, Stopeck A, Outwater E, Gillies RJ (2003) Correlation of chemotherapeutic response with the apparent diffusion coefficient of water (ADCw) of liver metastases. Proc Int Soc Magn Reson Med 11: 533

Therasse P, Arbuck SG, Eisenhauer EA, Wanders J, Kaplan RS, Rubinstein L, Verweij J, Van Glabbeke M, van Oosterom AT, Christian MC, Gwyther SG (2000) New guidelines to evaluate the response to treatment in solid tumors. J Natl Cancer Inst 92: 205-216

Tofts PS (1997) Modeling tracer kinetics in dynamic Gd-DTPA MR imaging. JMRI J Magn Reson Imaging 7: $91-101$

Toms JR (ed) (2003) CancerStats Monograph. London: Cancer Research UK Wasser K, Klein SK, Fink C, Junkermann H, Sinn HP, Zuna I, Knopp MV Delorme S (2003) Evaluation of neoadjuvant chemotherapeutic response of breast cancer using dynamic MRI with high temporal resolution. Eur Radiol 13: $80-87$ 
Weber WA (2005) Use of PET for monitoring cancer therapy and for predicting outcome. J Nucl Med 46: $983-995$

Zhao M, Pipe JG, Bonnett J, Evelhoch JL (1996) Early detection of treatment response by diffusion-weighted H-1 NMR spectroscopy in a murine tumour in vivo. Br J Cancer 73: 61-64
Zhou R, Pickup S, Yankeelov TE, Springer CS, Glickson JD (2004) Simultaneous measurement of arterial input function and tumor pharmacokinetics in mice by dynamic contrast enhanced imaging: effects of transcytolemmal water exchange. Magn Reson Med 52: $248-257$ 